\title{
Machine Learning Based Autonomous Road Maintenance System Using Cold Lay Asphalt
}

\author{
${ }^{1}$ Abhinav Sreesan, ${ }^{2}$ Anirudh Shankar, ${ }^{3}$ Vignesh Vaidyanathan, ${ }^{4}$ Shubhangi Kharche \\ Department of Electronics and Telecommunication Engineering, SIES Graduate School of Technology, Navi Mumbai, India \\ Email: abhinav.abhinav@siesgst.ac.in,shankar.anirudh@siesgst.ac.in,vignesh.vaidyanathan@siesgst.ac.in, shubhangi.kharche@siesgst.ac.in
}

\section{Received: 09 ${ }^{\text {th }}$ July 2018, Accepted: $14^{\text {th }}$ August 2018, Published: 31 ${ }^{\text {st }}$ August 2018}

\begin{abstract}
Road maintenance is quotidian problem faced by the concerned authorities as it requires time, resources and labor and if not treated with utmost alacrity, may result in mishaps and accidents. The following paper suggests an innovative approach that can reduce the time and labor requirement by incorporating concepts of image processing and machine learning with cutting edge materials like Cold Lay Asphalt to give an efficient solution to the afore mentioned problem. The primary goal of this paper is to identify and highlight an application of autonomous vehicles. To this end, the following paper proposes a machine learning based autonomous road maintenance system (ML-ARMS). The vehicle (Bot) in ML-ARMS is trained and controlled using Raspberry-Pi. The accuracy of the Machine Learning algorithm for 500 training images is found to be $42.778 \%$.
\end{abstract}

Keywords: Autonomous Vehicle, Cold Lay Asphalt, Road Maintenance.

\section{Introduction}

Road maintenance has been a tedious task especially in a country like India with over hundred thousand kilometres of roadways that have to be constantly maintained. Every year a major part of the Government's budget goes towards the maintenance of these roads. Thus, with advancements in vehicle automation technology it becomes possible to automate the process and to have a completely independent system that can be monitored remotely; also new road surface materials have made it possible for a quick and efficient maintenance process. This reduces a boatload of money spent in road maintenance and also provides scores of employment opportunities in the field of science and technology. The proposed system used for the maintenance of the damaged/faulty roads has sensors fitted on-board the vehicle which accurately detects the aberrations on the surface and then provide remedies by cleaning it and resurfacing the area to provide a smooth and clean surface for traversing. This will be extremely beneficial to avoid road accidents and won't proliferate traffic in that area. Now, for the development of this model ultrasonic distance sensors and cameras have been used to accomplish the proposed model. A vehicle is built which runs autonomously with the assistance of camera and two sensors to detect obstacles, road signs \& signals. Sensors are attached to the bottom of the vehicles to detect abnormalities of the road so as to fix it. A special kind of road surfacing material called cold lay asphalt has been developed recently which can be used to cure the roads and make sure that the roads are smooth enough for traversing. The cold lay asphalt is a type of mixture analogous to that of concrete as it doesn't need any type of heating and is used at variety of temperature range. The material is a lot like conventional asphalt as it takes the shape of the potholes just after dispensing the material.

The paper is structured to discuss the related work in section II, The Autonomous Road Maintenance System in section Methodology in section IV; Results in section V; Conclusion $\&$ Future Scope in section VI followed by References.

\section{Related Work}

Shuaishpauai Yang et. al. [1] has proposed a kinematic model based real time path planning algorithm. The algorithm considers all the constraints including kinematic constraints and obstacles; represents them using polynomial parameter and generates an AA guide line using the Bezier curve. Emilio Frazzoli et. al. [2] has seconded that with a model which proposes a randomized path planning architecture for dynamic systems in the presence of both considerations fixed and moving obstacles. This architecture considers the dynamic constraints on the motion of the vehicle and provides a consistent decoupling between low-level control and motion planning. The concept of Autonomous vehicles, that can sense its environment and navigation without human intervention dates to the 1930s, but the first true attempt of an autonomous vehicle came in the 1980s when Carnegie Mellon University's Navlab [3] used a Warp supercomputer for navigation and removal of obstacles. Image Processing is a salient concept used for the working of the autonomous vehicles all over the world. Detection of sign boards, pedestrians, vehicles that are approaching are done using the image processing. As the images captured might not be lucid, there arises a need for another image processing technique to get an accurate image without obscurities. Hardik Modi et. al [4] demonstrated image processing capabilities of the Raspberry Pi processor with the sensitivity of a motor driver circuit to attain precise and accurate motion of the vehicle. A CCD camera was used to harness the capabilities of image processing algorithms that were applied to obtain the object from the background of the image. Kenshiro Hashimoto et al. [5] has also demonstrated the utility of image processing to detect lane markings using Sobel filter and the Hough transform module and then triumphed in altering the trajectory of the vehicle. Richard Brunauer et al. presents a prototypical implementation of a road surface condition monitoring system that analysed the vertical acceleration signal from vehicle-mounted accelerometer sensors (physical jerks). His work proposes a linearly referenced road surface condition 


\section{Helix Vol. 8(5): 4002- 4006}

index (RSCI), being derived from position and measurements of the accelerometer and can catalogue vehicle-sensed road surface quality. A road operator supported field trial using a prototypical system for raw data collection, analysis and integration of the derived surface condition information into the road operator's legacy systems was conducted to collect sample data along with field experiences and to learn about the benefits of such an approach by considering it in the perspective of road operators. Autonomous vehicles are being utilised only for transportation purposes and very limited industrial applications and the autonomous vehicles for maintenance of roads has been slept upon. Apart from this existing road surfacing materials including asphalt and concrete have extensive curing periods and are also labour intensive. Cold lay asphalt [7] is a road material that is obtained by blending bitumen emulsion, aggregate, water and cement at ambient temperature. Tahseen Saadoon et.al. [8] has validated Cold asphalt mixture (CAM) which is a complex visco-elasto-plastic material that can be implemented for construction of roads at the wide range of temperatures. CAM is extremely effective in case of heavy traffic roads, where the poor quality of the concrete tends to generate higher amount of road fracture under traffic loads. Hence it has become extremely pivotal that asphalt layers adapt to such deformations without cracking.

Raspberry Pi based image processing used by Hardik Modi [4] and Kenshiro Hashimoto [5] has been used in our system to detect the edges of the road surface. Cold lay Asphalt Material (CAM) has been used for surfacing roads manually but an autonomous solution for the same is implemented in the system. The related work includes methods for making a self-driving car using image processing, method to find defects in the roadways, and a method to fix the roads without using extensive heating machinery. The objective of the existing work was to develop a way for detecting and locating damages on the roads by a self-driving bot and fixes the damage without need of multiple machinery and heat curing. To meet these objectives, a new approach, where a self-driving bot scans the road for damages and fix them using a material called cold lay asphalt. The objective of the existing work was to develop an autonomous vehicle which can its route and drive itself. The road fixing material developed called Cold Lay Asphalt for fixing roads needs no curing. The proposed work in this paper merges the application of the fore mentioned system using which the vehicle becomes autonomous and at the same time can detect the defects/damages on the road and take corrective measures.

\section{Autonomous Road Maintenance System}

\section{A. System Description}

The brain of the system is the Raspberry Pi 3 Board as it supports heavy image processing capabilites and has support for OpenCV libraries to be used to process the image. It is interfaced with a PiCamera v1.0 module that is used to collect the images for the manoeuvring of the bot. It is also interfaced with an Ultrasonic Distance Sensor for detecting obstacle in its path. An Infrared Photodiode array has been used to detect potholes on the surface of the road, a servo motor based dispensing mechanism for cold lay asphalt as depicted in the system design in Fig. 1 and the list of components is described in Table 1. The bot is actuated using four 300rpm geared DC Motors which is controlled by an L298 dual $\mathrm{H}$ bridge motor driver and the motors are powered by a $2200 \mathrm{mAh}$ Lithium Polymer battery.

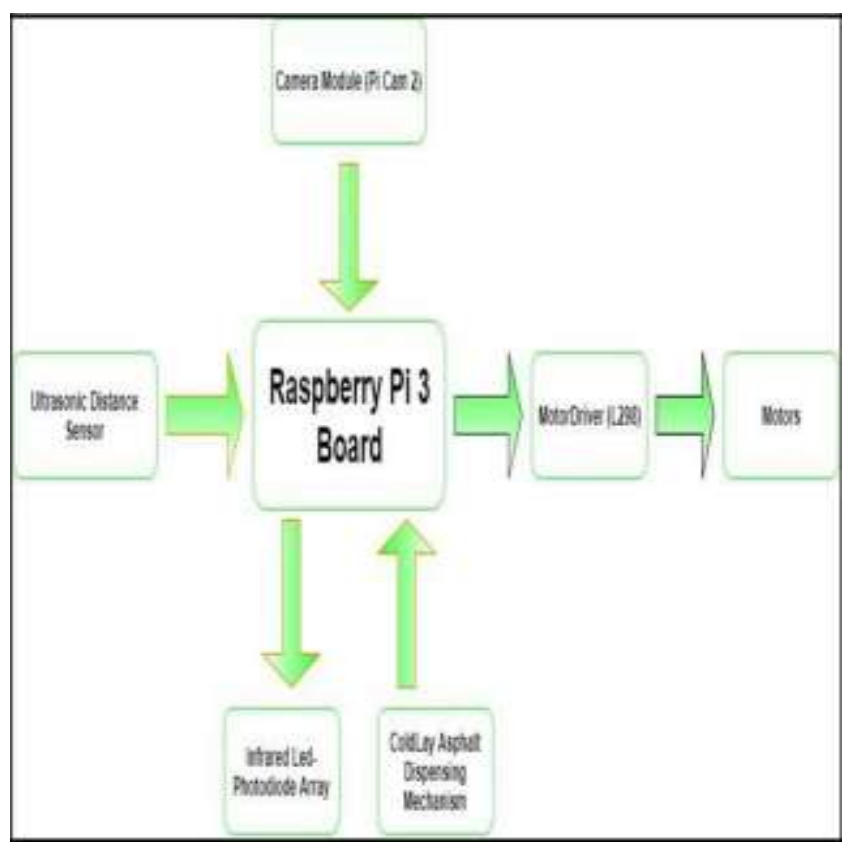

Fig. 1: The Proposed System [ML-ARMS]

\author{
Hardware \\ Pi Cam 2 \\ Servo Motor \\ Atmega 328 \\ Distance Sensors \\ LM298 Motor Driver \\ Software \\ Python 2.7 \\ OpenCV Library \\ NumPy Library
}

Raspberry Pi 3 Board

\section{Methodology \\ A. Artificial Neural Networks}

Artificial Neural Networks ANN, are a class of system that were inspired by the biological neural networks which was vital in the development of animal brains. The main benefit of using neural network is that it only requires to load the trained parameters after training, making predictions very fast. The other side of the input image is used for teaching and for predictions. There are $38,400(320 \times 120)$ nodes in the input layer and 32 hidden layer nodes. The hidden layer nodes are chosen arbitrarily. There are four nodes in the output layer where each node is used to steer the vehicle in all the directions: left, right, forward and reverse respectively. 


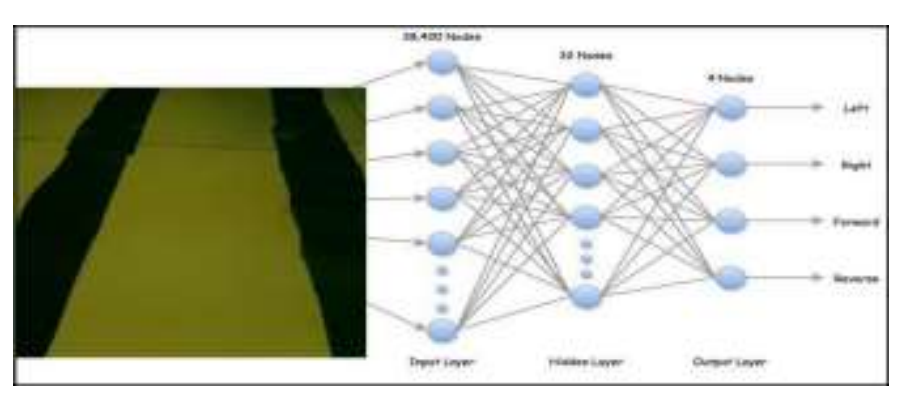

Fig. 2: Neural Networks

Based on the prediction made by the MLP algorithm, and the distance sensor values the bit is maneuvered to navigate the road in an autonomous manner as depicted in Fig. 2.

All the neurons in Multi-Layer Perceptions are similar. Each neuron has several input links and several output links. The values obtained from the earlier layers are summed up with certain weights and added to the bias term. The transformation of sum is done for different neurons.

\section{B. Multi-Layer Perceptron}

A perceptron is a linear classifier and are also called as an algorithm that sorts the input by dividing it into two groups with a straight line. Input is typically a vector $\mathrm{x}$ multiplied by weights $\mathrm{w}$ and added to $\mathrm{a}$ bias $\mathrm{b}$ and is given by the below equation:

$$
\mathbf{y}=\mathbf{w} * \mathbf{x}+\mathbf{b}
$$

A multilayer perceptron (MLP) is a deep, artificial neural network consisting of multiple perceptrons. As the name suggests they are made of an input layer to receive the signal and an output layer that forecasts about the input, and in between them, an arbitrary number of hidden layers that are the true computational engine of the MLP. Usually MLPs with one hidden layer can approximate any continuous function. Multilayer perceptions are frequently applied to supervised learning problems. They usually train on a set of input-output pairs to model the correlation between the two. Training involves adjusting the parameters, weights and biases, of the model to minimize error. Back propagation is used to make the weight and bias adjustments comparable to the error, and the error itself can be measured in many different methods, one such method is root mean square error (RMSE). In MLP algorithms the Epoch is first initialized with the value one, the weights and biases are assigned with random values. Next the input patterns are acquired to compute the output values. The mean square error is calculated and checked with predefined values to decide when to stop the training network. At the end of each iteration of the cycle as depicted in Fig. 3, the weights and biases are updated and the process is repeated until one of the two conditions required to stop the training network is satisfied. Using the MLP algorithm a hidden layer with 32 nodes is defined which has 4 output nodes one for each direction. In the input layer arrays are generated from the image matrix. The matrix is then compared with existing models to provide an output at the output layer that is responsible for the navigation of the vehicle. The same method is used to train the vehicle to detect other parameters like road sign detection and traffic signal detection by generating classifiers and the vehicle autonomous. Using the MLP algorithm a hidden layer with 32 nodes is defined which has 4 output nodes one for each direction. In the input layer arrays are generated from the image matrix. The matrix is then compared with existing models to provide an output at the output layer that is responsible for the navigation of the vehicle. The same method is used to train the vehicle to detect other parameters like road sign detection and traffic signal detection by generating classifiers and the vehicle autonomous.

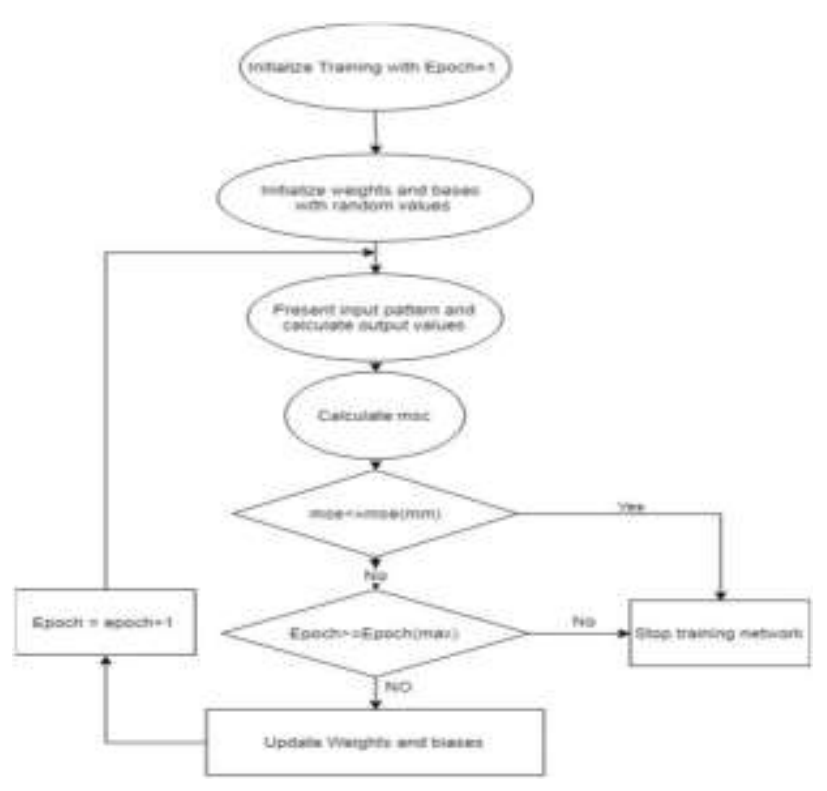

Fig. 3: Flowchart for MLP Training Algorithm

\section{DISTANCE MEASUREMENT}

The geometrical representation of the method used to calculate distance using monocular vision in depicted in the Fig. 4 where the distance $\mathrm{d}$ represents the distance of the camera from the object and $\mathrm{h}$ is distance of the camera form the ground.

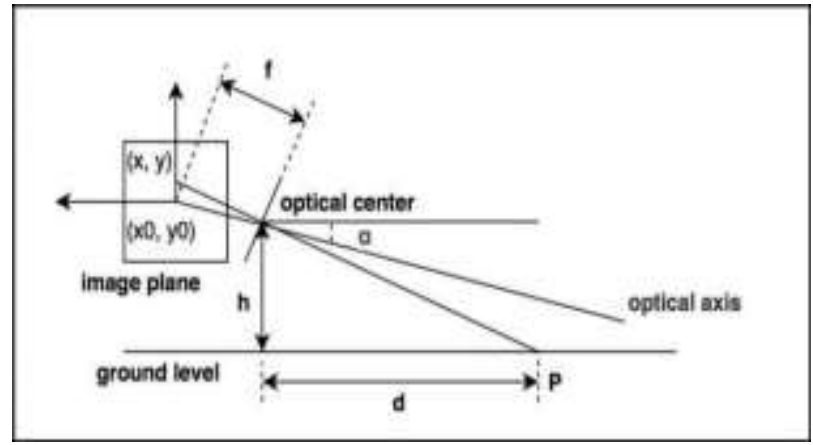

Fig. 4: Diagram of the Vision of Pi Camera [9]

Here we calculate the distance from the object to be tracked by using the principle of triangle similarity. First, we take an image of the object at a known distance and measure the width of the object in pixels. Then as we move the camera towards and away from the object we can calculate the distance using triangle similarity. First the focal length of the camera was computed by using the formula 


$$
\mathbf{F}=(\mathbf{P} \times \mathbf{D}) / \mathbf{W}
$$

Here $\mathrm{F}$ is the perceived focal length, $\mathrm{W}$ is the known width of the object, $\mathrm{D}$ is the distance from the object, $\mathrm{P}$ is the apparent width of the object in pixels. Next we can calculate width of unknown objects using the relation with unknown distance D'

$$
\mathbf{D}^{\prime}=(\mathbf{W} \times \mathbf{F}) / \mathbf{P}
$$

The main recognition of this technique is that it enables us to get the distance from the objects like traffic signal, road signs and turns in the road, to enable the vehicle to make decision according to the prediction given by the MLP algorithm.

\section{Cold Lay Asphalt:}

Cold Lay asphalt a road resurfacing substance is a blend of bitumen emulsion, aggregate, water and cement at ambient temperature. It helps to ameliorate social, economic and environmental perquisites over variant tarmac materials, such as hot mix asphalt, etc.

Development: Cold Asphalt amalgam is a bituminous materials incorporated by cold aggregates with an asphalt emulsion and water. Unlike Hot Mix Asphalt CAM can be produced at low temperatures. Therefore, the use of CAM more economically and financially viable, while reducing the pollution and occupational hazards. As far as the ecological and financial reverberations are considered, CAM can be refined by developing it with $100 \%$ recycled materials from Construction and Demolition Waste Aggregates.

\section{Results and Discussions}

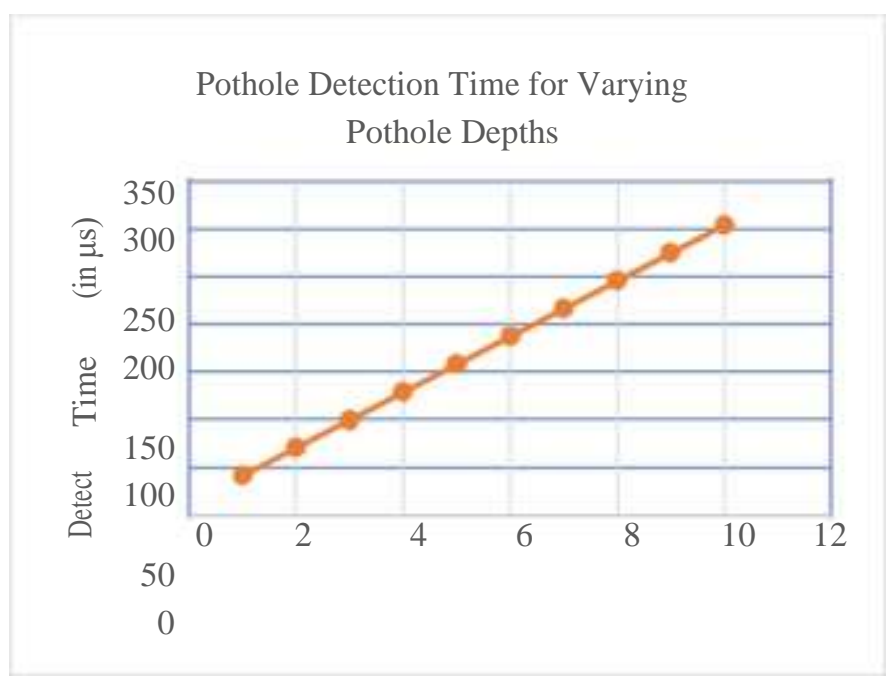

Depth of Pothole (in $\mathrm{Cm}$ )

\section{Fig. 5: Graph for Pothole Detection Time for varying Depths}

The graph in Fig. 5 depicts that as the distance between the senor $\&$ the surface of the road increases it is observed that there is an increase in the detection time. But as the time delay is of the order of microseconds it is within the margin allowable for efficient detection and filling of potholes

A. Image detection time and accuracy

The motor speed of the motors used in the system is $300 \mathrm{rpm}$. This speed is slower than the speed of conventional vehicle but to allow the raspberry pi to process the image in real time the speed of the boot should be kept within this limit. In real world application a more powerful processor is used that can process images faster to enable the vehicle to move faster.

Accuracy of MLP Training algorithm

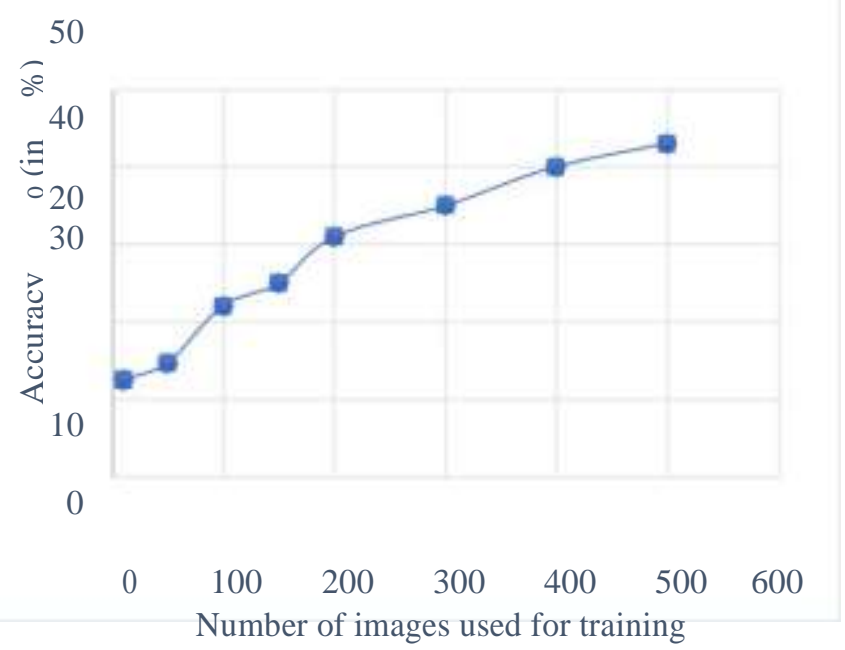

Fig. 6: Graph for MLP Training algorithm accuracy for varying number of images used for training. The graph in Fig 6 depicts that as the number of images used to train the MLP algorithm increases the obtained accuracy increases. The accuracy defined here refers to the percentage of correct predictions made per total number of predictions made. Also, we can collect images even while the vehicle is running and use the images to improve the MLP algorithm to get a better and safer autonomous vehicle.

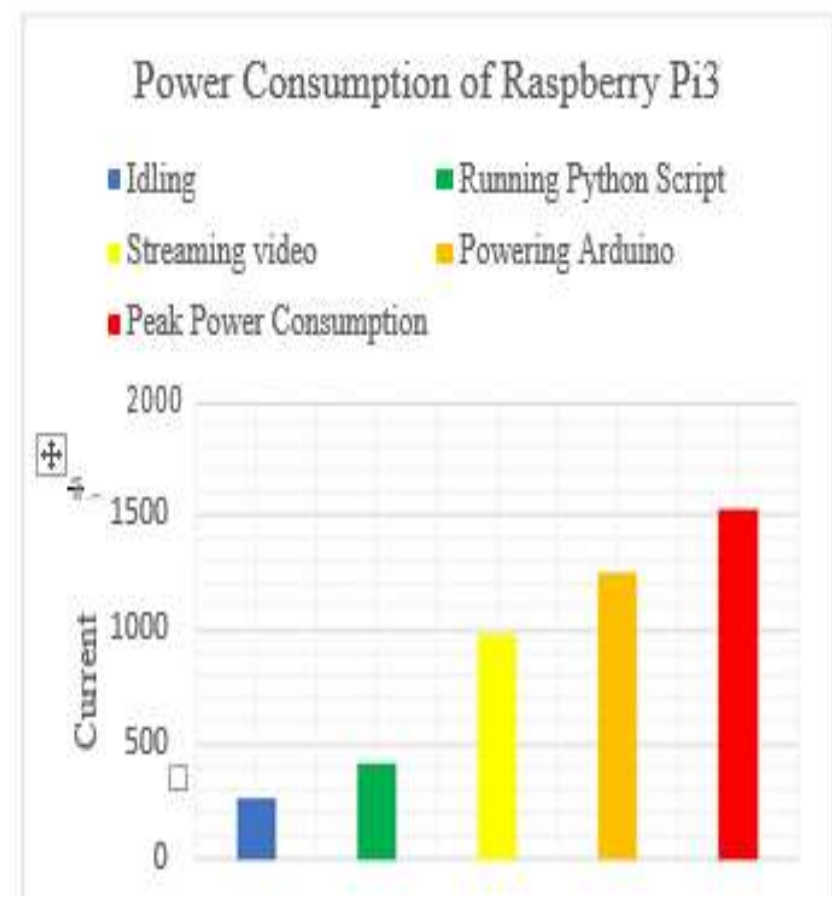

Fig. 7: Graph for Power Consumption of Raspberry Pi3 
The graph in Fig. 7 shows the variation of current consumption of the Raspberry Pi 3 board under various load conditions. From the above result it is concluded that even under high load the total current required is within $2 \mathrm{~A}$ and thus a small battery can be used to power the board.

B. Comparison between MLP and EBF Algorithms

MLP(Multi-Layer Perceptron) uses dot products (between inputs and weights) and sigmoidal activation functions (or other monotonic functions such as REL) and training is usually done through back propagation for all layers (which can be as many as you want).

RBF (Radial Basis Function): uses Euclidean distances (between inputs and weights, which can be viewed as centers) and (usually) Gaussian activation functions (which could be multivariate), which makes neurons more locally sensitive. Thus, RBF neurons have maximum activation when the center/weights are equal to the inputs

As the application of Neural Network in this system requires a supervised algorithm with two hidden layers, MLP algorithm has been used.

C. Time for dispensing cold lay asphalt

The time required for the opening of the servo motor to dispense the cold lay asphalt is 500 milliseconds. The dispensing time varies according to the size of the pothole and the volume of the pothole.

\section{Conclusion and Future Scope}

This proposition is a completely autonomous vehicle capable of traversing the streets; it can detect instructions on the road and traffic lights using image processing and can detect hurdles in its path using an array of ultrasonic sensors and react accordingly. Aberrations on the tarmac are detected using an array of IR Photodiodes and then the Asphalt Material is dispensed on the area thus making the road smooth and pothole free. With progress and innovations in this concerned field, the price incurred on such a vehicle can be brought down which will enable them to be widely used. The system can then be upgraded to a mesh of connected vehicles that can communicate in real time to improve speed and efficiency of the task. Machine learning algorithms have increased the proficiency of such cars. More advanced technology like LIDAR can be implemented to enable the vehicle to traverse in places with rougher terrain.

The system with small modifications can be implemented in a variety of other application so as to automate daily routines like an autonomous garbage collection vehicle or an autonomous road laying vehicle. It can also be used in the industrial sector to perform tasks in hazardous environments. The ML-ARMS if adopted on a wider scale will benefit the society and will render accidents due to road conditions obsolete. Further developments can be made to the proposed system to implement a completely autonomous road laying and monitoring system that can be implemented in laying roads in remote and rural areas without the need for any human intervention.

\section{References}

[1] Shuaishuai Yang, Zhuping Wang and Hao Zhang. Kinematic model based real-time path planning method with guide line for autonomous vehicle. ControlConference (CCC), 2017 36th Chinese, Dalian, China, 2017.

[2] E. Frazzoli, M.A. Dahleh and E. Feron. Real-time motion planning for agileautonomous vehicles. American Control Conference, 2001. Proceedings of the 2001.

[3] "Navlab", https://www.cs.cmu.edu/afs/cs/project/alv/

[4] Hardik Modi, V. Shastri Nirav, H and Shastri Malav. Obstacle Avoidance System in Autonomous Ground Vehicle Using Ground Plane Image Processing and Omnidirectional Drive. Computing Communication Control and Automation (ICCUBEA), Pune, 2015.

[5] K. Hashimoto, S. Nakayama, T. Saito, S. Ishida, K. Unuora, N. Oono, Y. Okada and J. Ishii. An image processing architecture and a motion control method for an autonomous vehicle. Intelligent Vehicles '92 Symposium, Detroit, MI, USA,July 1992..

[6] Richard Brunauer and Karl Rehrl. Supporting road maintenance with in-vehicle data: Results from a field trial on road surface condition monitoring. Intelligent Transportation Systems (ITSC), 2016 IEEE 19th International Conference,2015.

[7] "Cold Lay Asphalt", https://www.tarmac.com/media/959003/product-datasheet-cold-lay-macadam-april-2017.pdf.

[8] Tahseen Saadoon, Alvaro Garcia, Breixo and GmezMeijide. Dynamics of water evaporation in cold asphalt mixtures. Nottingham Transportation Engineering Centre [NTEC], Department of Civil Engineering, University of Nottingham,Nottingham NG7 2RD, UK, May 2017

[9] "https://zhengludwig.wordpress.com/proje=cts/selfdriving-rc-car/" 